Rev. SINAPSIS, Edición № 12, Vol. 1, Diciembre 2018

ISSN 1390 - 9770

\title{
La drogadicción y su impacto en las familias
}

\section{Drug addiction and its impact on families}

Elvira Geoconda Villacreses Veliz M.Ge. ${ }^{(1)}$

Aida Monserrate Macías Alvia M.Gs. ${ }^{(2)}$

Carmen Mercedes Erazo Mero Lcda. ${ }^{(3)}$

Sonia Patricia Ubillús Saltos. Ph. D. ${ }^{(4)}$

(1), (2) y (3) Universidad Estatal del Sur de Manabí, Jipijapa, Ecuador

(4) Instituto Tecnológico Superior "Portoviejo. Manabí. Ecuador

Contacto: yoqui.rovi.2010@ hotmail.com

Receptado: 26/01/2018 Aceptado: 16/04/2018

\section{Resumen}

El trabajo de investigación "La drogadicción y su impacto en las familias de la ciudadela Nueva Esperanza" planteó como objetivo: determinar de qué manera la drogadicción incide en las familias de la ciudadela Nueva Esperanza. Los métodos utilizados fueron: el descriptivo y la observación. La población universo la constituyen 200 familias, de las cuales la muestra son 50 familias y 10 dirigentes

del sector, a las primeras se les aplicó encuestas y a los segundos entrevistas. La evaluación de los resultados indica que en la ciudadela Nueva Esperanza entre un 20 a $25 \%$ de la población joven masculina y entre el 10 y $12 \%$ de adultos varones consume algún tipo de droga; las drogas más utilizadas son la marihuana y la cocaína, las mismas que están causando problemas psicológicos, económicos, desintegración familiar y otros conflictos, ya que los consumidores presentan cambios como comportamiento agresivo e incluso hay una potencial inclinación por el robo. La presencia de autoridades no se ha visualizado por la comunidad. Estos resultados obtenidos conducen al 
Rev. SINAPSIS, Edición № 12, Vol. 1, Diciembre 2018

ISSN $1390-9770$

planteamiento de la propuesta de implementación de un modelo de gestión de prevención, control y erradicación del consumo de droga en la ciudadela Nueva Esperanza.

Palabras clave: conflictos, desintegración, comunidad, comportamiento.

\begin{abstract}
The Research Project "Drug addiction and its Impact on Families of the citadel Nueva Esperanza" wont As Objective: S. determine how the drug affects the citadel Families New expect. Son Methods Used: descriptive and scientific observation. The world population is constituted 200 families, of which the simple is 50 families and 10 leaders of the sector, First Apostolic them Seconds Surveys and Interviews. The evaluation of the results indicates that the citadel Nueva Esperanza UN Between 20-25\% of young men and Population Between 10 and 12\% Adult Male ANY type of drug consumption; More Drug son used marijuana and cocaine, which are causing Same Psychological Problems, Economic, Family Disintegration OTHERS AND Conflict and Consumer Present That Changes As aggressive behavior and even a potential Tilt BY theft. The presence of authorities is not displayed by the community. These results Lead to approach m of Proposed Implementation of the UN Model Management Prevention, Control and eradication of drug consumption in the citadel Nueva Esperanza.
\end{abstract}

Keywords: conflicts, disintegration, community, behavior.

\title{
Introducción
}

El uso y abuso de las drogas ilegales se ha convertido en un problema universal que afecta directamente al grupo familiar ocasionando diferentes conflictos internos que pueden producir serios problemas que darán inestabilidad al núcleo familiar, así lo demuestran diferentes estudios realizados al respecto, como se manifiesta "las crisis familiar, no implican sólo la vivencia de circunstancias negativas o la máxima expresión de un problema; sino también la oportunidad de crecer y superar las contradicciones" las oportunidades de desarrollarse como seres humanos en un núcleo familiar se disminuyen cuando los conflictos son provocados y controlados por las adicciones. (Herrera, 2012) 
Rev. SINAPSIS, Edición № 12, Vol. 1, Diciembre 2018

ISSN 1390 - 9770

La Oficina de las Naciones Unidas contra las drogas y el delito señala que hay una estimación de entre 99.000 a 253.000 personas que murieron en el 2010 a consecuencia del consumo de drogas ilícitas en el mundo, lo que significó entre el 0,5 y el 1,3\% de la mortalidad universal de la población de 15 a 64 años de edad. El consumo de drogas representa además un problema de índole económico para las sociedades del mundo, en este sentido el estudio citado indica que son necesarios de 200.000 a 250.000 millones de dólares, el 0,3 al 0,4 \% del PIB del planeta, para asistir el tratamiento a los asuntos de droga, lo que lógicamente tiene una repercusión directa en la familia.

Entre los problemas que han contribuido al aparecimiento y aumento del uso de drogas en la mayoría de países, están las regresiones económicas, ingresos percapita mínimo, la pobreza, la dificultades demográficas, el crecimiento de las migraciones hacia los centros urbanos que acarrea el déficit en los servicios básicos, en la calidad de la educación y la salud y también un progresivo resquebramiento en la estructura familiar. Todo esto obedece más a cambios políticos y socioeconómicos que a los hábitos culturales y prácticas rutinarias. Tomando en cuenta que hay una contradicción entre el discurso penalizador y el que permite el consumo que han adoptado gobiernos de diferentes estados afectando así de muchas maneras a la salud de los seres que la consumen cotidianamente sin importar su raza, su sexo, su posición económica, su credo. (Romero S. M., 2018)

El Ecuador no ha estado ajeno a esta problemática y en el tema de droga ha ido a la par con la incidencia que este ha tenido en todos los países de América Latina, así lo demuestran diferentes estudios en donde persisten profundas distorsiones y una gran manipulación de algunos políticos que tienen cerrada mentalidad conservadora respecto a la cuestión de las drogas. El consumo diario de esta provocaba alteraciones en el sueño y pérdida del apetito, y si la persona no la consumía, podía entrar en un estado de desesperación, cambio de ánimo y muchas veces hasta el suicidio o producir efectos adversos por su consumo; todo lo antes mencionado por la falta de conocimiento y la poca cultura que tiene nuestra sociedad ante este tema. (Del Bosque, Fuentes, Diáz, \& Espinola, 2014)

Varias investigaciones han demostrado que existe una relación entre el consumo de drogas y una serie de variables del entorno familiar, entre las cuales se hallan los hábitos de consumo de drogas 
Rev. SINAPSIS, Edición № 12, Vol. 1, Diciembre 2018

ISSN 1390 - 9770

de los padres y el hecho de que en la familia no exista una figura materna; de igual relevancia es que en el seno del hogar el ambiente familiar sea hostil. En cuanto a los factores de riesgo, se ha encontrado que las medidas disciplinarias, la actitud favorable a la experimentación de sustancias y la deficiente comunicación entre los integrantes de la familia hacen muy probable que los jóvenes se involucren en el consumo de drogas y se desencadenen en una serie de conflictos que no se solucionaran con facilidad. (Araiza, Silva, Coffin, \& Jiménez, 2013)

Por otro lado el CONSEP, en su Plan de prevención integral de drogas, indica que las drogas y sus consecuencias se han convertido en uno de los temas más preocupantes para la sociedad ecuatoriana, lo que ha generado el trabajo coordinado de las autoridades competentes para la formulación participativa del mismo para el periodo 2012-2013 y que se actualiza luego para el lapso 2013 - 2017, más aún cuando ya el artículo 364 de la Constitución ecuatoriana, dice que las adicciones son un problema de salud pública y que corresponde al Estado desarrollar programas de prevención y control del consumo de alcohol, y sustancias estupefacientes y psicotrópicas. Adicionalmente, la Constitución prescribe que, en ningún caso, se permitirá la criminalización ni se vulnerarán los derechos constitucionales. También se señala la obligación de ofrecer tratamientos y rehabilitación a los consumidores ocasionales, eventuales y problemáticos. (Paladines, 2013)

Al analizar los factores asociados al consumo de drogas, se identificaron los siguientes: no ser estudiante de tiempo completo, no contar con un trabajo, tener un bajo nivel socioeconómico, ser estudiante de licenciatura, estar involucrado en un accidente de tránsito grave, el intento suicida, el abuso sexual, la impulsividad, tener una baja percepción del riesgo de consumir sustancias y la disponibilidad de éstas en el entorno. Además, tener amigos que consuman drogas, que toleren su consumo, que presenten actos antisociales y la falta de amigos con conductas prosociales, también son factores significativos para el consumo experimental o regular. En cuanto a la familia, la falta de involucramiento de los padres, el monitoreo negativo de parte de ellos y el consumo de drogas en la familia se asocian con el uso experimental o regular. (Villatoro, Mendoza, \& Moreno, 2014)

Informes del CONSEP señalan, que la prevalencia de vida (PV) de drogas ilícitas establece que el consumo de marihuana continúa siendo el más conocido en la población (4.3\%), superando a otras drogas que muestran prevalencias de vida bajas. Estos datos obedecen al hecho de que la marihuana 
Rev. SINAPSIS, Edición № 12, Vol. 1, Diciembre 2018

ISSN 1390 - 9770

es la droga ilícita más rápida de adquirir (23.1\%) antes que la cocaína (13.5\%) y la base (10.1\%). El consumo de drogas ilícitas causa una alta incidencia de agresividad física, verbal, abuso sexual y violencia intrafamiliar. (CONSEP, 2012-2013)

Estos datos se generalizan para todas las provincias del Ecuador, entre ellas la provincia de Manabí y en el caso particular de esta investigación la ciudadela Nueva Esperanza del Cantón San Vicente, en donde se ha evidenciado un notorio brote de consumo de drogas ilícitas, lo que consecuentemente motiva la realización de esta investigación, teniendo como problema principal la deficiencia en el conocimiento del consumo de la droga y su impacto en las familias de la ciudadela Nueva Esperanza del Cantón San Vicente.

Las drogas en sus diferentes manifestaciones han invadido nuestro entorno. Se trata de un fenómeno de múltiples facetas que incluye las etapas de producción, tráfico, distribución, consumo y dependencia; afectan a todos los grupos de la población y tienen implicaciones importantes para la salud pública de la población o del entorno que no rodea. El problema requiere de enfoques novedosos que permitan enfrentar un problema complejo y cambiante, que nos permita buscar soluciones a un problema muy complejo que ha venido evolucionando a lo largo del tiempo y que no hemos podido detener; por eso es importante poder determinar mediante esta investigación cuales son los factores que impactan y afectan a las familias de nuestro entorno. (Medina, Real, \& Villatoro, 2013)

Según la Organización Mundial de la Salud, droga es toda sustancia que, introducida en el organismo puede modificar la conducta del sujeto y provocar un impulso irreprimible a tomar la sustancia de forma continuada o periódica, a fin de obtener sus efectos, y a veces, de evitar el malestar de su falta. En sentido amplio, se trata de una sustancia que afecta las funciones corporales y la conducta. (Mosquera, 2011)

La familia: La familia sigue constituyendo el núcleo más importante de socialización, a pesar de los acelerados cambios sociológicos que se han producido durante las últimas décadas y que han supuesto una transformación de su estructura. La familia es referencia de vida de cada persona en nuestra sociedad; son estructuras complejas en donde se vierten las emociones de los individuos, son filosofías de vida en donde se mantienen los vínculos afectivos, valórales y en donde se ponen más a prueba los conflictos humanos. En el seno de la familia se producen procesos básicos: la 
Rev. SINAPSIS, Edición № 12, Vol. 1, Diciembre 2018

ISSN 1390 - 9770

expresión de sentimientos, adecuados o inadecuados, la personalidad del individuo y patrones de conducta; todo esto se aprende en la dinámica familiar y los que así aprendan enseñarán a su vez a sus hijos, más o menos del mismo. (Suárez, 2012)

Dentro de la familia se producen una serie de interacciones sociales que influyen en el curso del desarrollo psicológico de la persona. Pero, ¿Qué ocurre cuando hay problemas dentro de la familia?

Esta revisión nos planteamos analizar la relación entre la desorganización familiar y el consumo de sustancias psicoactivas. Sin embargo, nos encontramos con que el concepto de desorganización familiar es muy amplio, y aunque se ha intentado clarificarlo y conceptualizarlo en distintas ocasiones, todavía su definición es ambigua y poco clara. (Becoña \& Martínez, 2012)

Para algunos adolescentes, experimentar el consumo de drogas es rito necesario para atravesar la fase de independencia. Influenciados generalmente por pares, hacen uso de sustancia de manera ocasional y los adolescentes que presentan personalidad compleja pueden ser más sensibles a dependencia de drogas Por sus características evolutivas, como la búsqueda de identidad personal e independencia, alejamiento de los valores familiares y énfasis en la necesidad de aceptación por el grupo de iguales, la adolescencia se convierte en la etapa evolutiva con mayor riesgo de inicio del consumo de drogas. (Canales, 2012)

\section{Materiales y Métodos.}

Para recopilar la información de la presente investigación, se ha utilizado el método descriptivo, la observación científica y las técnicas de la encuesta dirigida a familias del sector y la entrevista aplicada a dirigentes de la comunidad objeto de la investigación. La población universo de este trabajo son 200 familias de la ciudadela Nueva Esperanza, de las cuales se ha tomado una muestra a través de una encuesta dirigida a 50 familias y una entrevista aplicada a 10 dirigentes comunitarios.

\section{Resultados}


Rev. SINAPSIS, Edición № 12, Vol. 1, Diciembre 2018

ISSN 1390 - 9770

Los resultados de este estudio reflejan datos similares a los que informa el CONSEP a nivel de país, ya que se identificó a la marihuana y la base de cocaína como las drogas que más se distribuyen y consumen en la ciudadela Nueva Esperanza del cantón San Vicente en la Provincia de Manabí, Ecuador, además también se corroboró que estas drogas ilícitas causan agresividad física y violencia inter e intrafamiliar. Es evidente que las acciones de autoridades competentes para contrarrestar el consumo de drogas en esta comunidad es inexistente en el conocimiento de las familias del sector y para el $90 \%$ de los dirigentes comunitarios. Por parte de la comunidad hay desconocimiento de la existencia de tratamientos para recuperación de la adicción a las drogas, mientras que para los dirigentes, este aspecto si es conocido.

Las consideraciones de estos resultados han permitido la formulación de un modelo de gestión para la prevención, control y erradicación de las drogas en la ciudadela Nueva Esperanza. Al preguntar con respecto a qué tipo de droga se consume en el sector, de las 50 personas encuestadas, 30 que representan el 60\%, manifestaron que es la marihuana, 10 que corresponden el 20\%, expresaron que es el alcohol, otras 10 que son el restante $20 \%$ manifestaron que es la cocaína. Al interpretar la lectura de estos datos, muestra que la mayoría de habitantes cree que la droga de mayor consumo en el sector es la marihuana, seguida con un $40 \%$ de diferencia del alcohol y la cocaína.

Cuando se preguntó a las personas del sector, en cuantas de cada 10 familias cree usted que hay un consumidor de drogas, 36 de los 50 encuestados que representan el 72\%, manifestaron entre 1 a 3 ; mientras que 13 que son el 26\%, han dicho que entre 4 a 8; y, finalmente 1 persona que es la representación del 2\% del universo de esta investigación ha dicho que entre 7 a 10.

Al analizar esta información es deducible que aunque la mayoría ha escogido la alternativa de menor incidencia, la situación no deja de ser preocupante y hasta cierto punto alarmante, pues en una comunidad en donde se identifica que en un promedio general en cada familia hay por lo menos un adicto a las drogas, es una comunidad que tiene muy comprometida negativamente, sus posibilidades de desarrollo y de mejoría de la calidad de vida, pues ya está infectada con este mal social.

Consultando sobre el tipo de problemas que ocasiona en el interior de la familia, el consumo de drogas, de las 50 familias encuestadas, 20 que son el 40\% del universo de la investigación, han dicho que si ocasionan problemas en el interior de la familia; 10 que son el 20\%, dicen que les 
Rev. SINAPSIS, Edición № 12, Vol. 1, Diciembre 2018

ISSN 1390 - 9770

ocasiona problemas psicológicos; y, 10 que viene a ser el 20\%, expresaron que esto trae problemas económicos, y seis que vendrían a ser el 12\%, dicen que hay desintegración familiar, y finalmente 4 que representan el $8 \%$ manifiesta que provoca otros problemas.

Se debe entender entonces que cuando en una familia hay un drogadicto, existen problemas, psicológicos, económicos, de desintegración familiar, y otros conflictos, se deduce entonces que la drogadicción afecta negativamente a la familia, ya que incluso llega a desintegrarla es decir aniquilarla, lo que es un problema realmente catastrófico, pues la familia es el núcleo de la sociedad y si la familia muere o se desintegra, la sociedad se enferma o pierde su rumbo.

Sobre si han presenciado o escuchado de algún conflicto interpersonal o interfamiliar en esta comunidad, 50 que es el 100\%, han dicho que si existe conflicto interpersonal o interfamiliar en esta comunidad como consecuencia del consumo de droga. Cuando los encuestados manifestaron que debido al consumo de droga se dan conflictos interpersonales e interfamiliares, se debe entender que existe una sociedad o comunidad potencialmente conflictiva, se deduce entonces, que hay que tomar medidas para prevenir y controlar el aumento del consumo de drogas y su erradicación, pues es evidente que estas prácticas están ocasionando una afectación al buen vivir que todas las familias del Ecuador merecen y que el Estado, así como la población son corresponsables de garantizarlo.

\section{Discusión}

De acuerdo a la investigación son necesarios nuevos estudios en esta temática con mayor análisis que permitan continuar profundizando sobre el fenómeno de las drogas en este grupo vulnerable y planificar y desarrollar estrategias que contribuyan a la efectiva inserción social de los adolescentes y sus familiares para mejorar los problemas tan comúnmente actuales como son la drogadicción.

La presente investigación es relevante ya que aporta a nuestra sociedad en la mejora y busca de soluciones al problema planteado buscando mejorar la calidad de vida de la sociedad. En la actualidad si se han realizado más estudios sobre el tema investigado, ya que es una problemática no solo a nivel local, sino a nivel mundial.

\section{Conclusiones}


Rev. SINAPSIS, Edición № 12, Vol. 1, Diciembre 2018

a) El consumo de drogas en la ciudadela Nueva Esperanza del cantón San Vicente, se da en la población joven y muy en particular la masculina, la que está más vulnerable para trajinar por el oscuro y perjudicial mundo de la adicción, este fenómeno obedece a la cultura de una sociedad machista en donde a los hijos se les está permitido permanecer más tiempo en la calle, en actividades de ocio con los amigos, lugar en donde van adquiriendo algunas influencias negativas, como es el caso del consumo de drogas.

b) Las drogas que más se distribuyen y consumen en la ciudadela Nueva Esperanza, son la marihuana y la base de cocaína, prevaleciendo según el criterio de las familias del sector, la marihuana, ante esta realidad, y aceptando el criterio del CONSEP cuando indica que la marihuana es la droga más fácil de obtener, podemos asimilar que probablemente esta facilidad para acceder está abriendo el camino para que la juventud masculina que es la que está más expuesta sea presa fácil de caer en la drogadicción.

c) Esta investigación estableció que en 3 de cada 10 familias hay una persona con adicción a las drogas en la ciudadela Nueva Esperanza, consecuentemente se concluye que está es una comunidad totalmente afectada por el consumo de drogas ilícitas, lo cual representa una situación digna de que las autoridades competentes le presten la debida atención.

d) La ciudadela Nueva Esperanza es una comunidad con un alto potencial conflictivo, lo cual afecta la convivencia familiar, comunitaria y el desarrollo turístico de la zona de influencia, esto es el Puente Los Caras y la Playa Los Perales, esta es una conclusión a la que se llega luego de observar los resultados de esta investigación, las familias encuestadas han expresado que el consumo de drogas provoca efectos de cambios de comportamientos que incluyen conductas agresivas e inclinación por el robo, en quienes la están consumiendo en el sector.

e) Las tres cuartas partes de las familias de esta población, desconoce de la existencia de algún tipo de tratamientos para la recuperación de los adictos a las drogas, pero hay una cuarta parte que si conoce de la existencia de estos tratamientos; mientras que para la totalidad de los dirigentes entrevistados, este tema no es ajeno. Se concluye que estos datos pueden ser aprovechados positivamente.

\section{Referencias}

Angelica, V. L. (2010). Estrategias de aprendizaje. Gondola, 4. 
Rev. SINAPSIS, Edición № 12, Vol. 1, Diciembre 2018

ISSN 1390 - 9770

Antonio, M. M. (2010). ¿Al Final, Qué es prendizaje Significativo? Qurriculum, 2.

Aparici, R. (01 de Mayo de 2011). Organizaciones de los Estados Americanos. Obtenido de La Educación: http://www.educoas.org/portal/laeducacion2010

Araiza Cárdenas Vanessa, S. R. (2013). Hábitos de estudio, ambiente familiar y su relación en el consumo de drogas en estudiantes. Psicología y Salud, 19(N²).

Ausubel, D. (01 de Agosto de 1983). Psicología Educativa y el labor docente. En D. D. Ausubel, \& O. L. Castiblanco abril (Ed.), Teoria del Aprendizaje Significativo (Vol. 5, pág. 1). Mexico: Index. doi:Pp27-37

Becoña Elisardo, M. U. (Julio-Septiembre de 2012). ¿Cómo influye la desorganización familiar en el consumo de drogas de los hijos. Adicciones, $24\left(\mathrm{~N}^{\circ} 3\right)$.

Cacheiro, M. (01 de Julio de 2011). Revistas de Medios y Educación. Obtenido de Revistas de Medios y Educación: http://acdc.sav.us.es/pixelbit/images/stories/p39/06.pdf

Caiza, M. (9 de Junio de 2012). Repositorio digital UCE. Obtenido de Incidencia de la atención dispersa en el aprendizaje: http://www.dspace.uce.edu.ec/handle/25000/253

CONSEP. (2012-2013). La prevención del uso y consumo de drogas desde la visión integral.

Del Bosque Jesús, F. A. (Sep/Oct de 2014). La cocaina: consumo y consecuencias. Scielo, $37\left(N^{\circ} 5\right)$.

Del Moral, M. E., \& Villalustre, L. (2012). Didáctica Universitaria en la era 2.0: Competencias Docentes en Campos Virtuales. Revista de Universidad y Sociedad del Conocimiento volumen $9,4$.

Dra., R. S. (2018). La Salud del Adolescente y del Joven. ARS Medica - Revista de Ciencias Médicas.

Gregoria Francisca, C. Q. (Noviembre de 2012). CONSUMO DE DROGAS PSICOACTIVAS Y FACTORES DE RIESGO. Cuidarte. 
Rev. SINAPSIS, Edición № 12, Vol. 1, Diciembre 2018

ISSN 1390 - 9770

J., M. (2011). Análisis de las tendencias de consumos de drogas de abuso e impactos en la salud del indiviuo en países de américa latina. Bogota.

Jorge, P. (Julio de 2013). Ni enfermos ni delincuentes . Revista Institucional de la Defensoría Publica del Ecuador, 4.

Medina Mora María, R. T. (Enero/Febrero de 2013). Las drogas y la salud pública: ¿hacia dónde vamos?. Scielo, 55( $\left.\mathrm{N}^{\circ} 1\right)$.

Metabase de Recursos Educativos. (2015). Teorías psicológicas del aprendizaje. Mexico: eUAEM.

Moreira, M. (2012). La Teoría del Aprendizaje Significativo Crítico: un r3eferente para organizar la enseñanza contemporanea. Revista Iberoamericana de Educación, 4.

O., S. (2012). La familia como eje fundamental en la formaación de valores. Venezuela.

O., S. (2012). La Familia como eje fundamental en la formación de valores. Venezuela.

Palomares, A. (2011). El modelo docente universitario y el uso de nuevas metodologías en la enseñanza, aprendizaje y evaluación. Revista de Educación, 3. Obtenido de Revista de Educación: http://www.revistaeducacion.educacion.es

Patricia, H. S. (Agosto de 2012). Factores de riesgo para la salud familiar: acontecimientos significativos. Scielo, $12\left(\mathrm{~N}^{\circ} 2\right)$.

Rivero, I., Gómez, M., \& Abrego, R. F. (2013). Tecnologías Educactivas y Estrategias Didácticas: Criterios de Seleccción. Educación y Tecnología, $N^{\circ}$ 3, 191.

Rivero, I., Marcela, G., \& Abrego, R. (2013). ecnologías educativas y estrategias didácticas: criterios de selección. Revista Educación y Tecnología, 3.

Romero, M., \& Quesada, A. (2014). Nueva tecnología y el Aprendizaje Significativo de la Ciencia. Dipósit Digital de Documents de la UAB, 2. 
Rev. SINAPSIS, Edición № 12, Vol. 1, Diciembre 2018

Salinas, J., Darder, A., \& Benito, B. d. (2011). Los mapas conceptuales como organizadores del proceso enseñanza aprendizaje: los itinerarios de aprendizaje. Investigación Novación Educativa i socioeducativa., 5.

Tunnermann, C. (2011). El Constructivismo y el Aprendizaje de los Estudiantes. Red de Revistas Científicas de América Latina, el Caribe, España y Portugal, 3 a 13.

Vera, A., \& Luis, V. (2011). Estrategias utilizadas por los docentes para promover el aprendizaje de la biología. Dialnet, 3 .

Villatoro Velázque Jorge, M. M. (sep/oct de 2014). Tendencias del uso de drogas . Scielo, $37\left(N^{\circ} 5\right)$. 